\title{
Surface Phonons
}

\section{RECENT RESULTS FROM COMPLEMENTARY PROBES}

\author{
J. Peter Toennies \\ Max-Planck-Institut für Strömungsforschung \\ Göttingen, Germany
}

\begin{abstract}
The 1992 Hewlett-Packard Europhysics Prize was awarded jointly to Professors G. Ertl (Fritz-Haber-Institut, Berlin), H. Ibach (Forschungscentrum, Jülich) and J.P. Toennies (Max-Planck-Institut für Strömungsforschung, Göttingen) for pioneering studies of surface structures, dynamics and reactions. Professor Toennies reports here on aspects of the cited research related to helium atom and electron energy loss spectroscopies.
\end{abstract}

Surface science continues to be a rapidIy growing area of research. From the chemical perspective, an important factor in this growth is a desire to understand the details of elementary chemical surface processes which form the basis of heterogeneous catalysis and corrosion. Interest in physical aspects comes from the realisation that surfaces become increasingly important as microelectronic devices shrink in size.

Much of the present research is devoted to establishing the exact structures of both clean as well as adsorbate-covered surfaces. In many crystals the structure at the surface is different from that in the bulk. For example, the interlayer spacing near the surface is often reduced by several percent (relaxation) and frequently the surface geometry is also different (reconstruction). One might suspect such changes to be simply due to the significant reduction in the number of nearest neighbours. However, there is now considerable evidence that the interatomic forces between surface atoms are in fact different than in the bulk. A knowledge of these forces is fundamental for understanding not only the lattice and the electronic structure but also elementary dynamical processes such as diffusion at surfaces. Although theory at this time cannot predict these forces, it is evident that there is a subtle interplay between the forces, structures and electronic states. At the surface, due to the loss of 3-d symmetry, this interplay is more delicately balanced than in the bulk.

\section{Surface Vibrations}

Within the last 10 years technological advances in $\mathrm{He}$ atom and electron energy loss scattering experiments have made it

J. Peter Toennies, Director of the Max-PlanckInstitut für Strömungsforschung, Bunsenstrasse 10, W-3400 Göttingen, has been an Associate Professor in the Physics Department, University of Göttingen, since 1971. He received his first degree from Amherst College, $M A$, USA, and a Ph.D. in chemistry from Brown University, RI, USA, in 1957. He then joined Bonn University's Physics Department, being awarded his Habilitation in 1965 and holding Visiting Professorships at the University of Florida, USA, and Chengdu Univ., PRC. possible to measure the small energy changes of several meV $\left(10^{-3} \mathrm{eV}\right)$ accompanying the excitation of surface vibrations. The vibrational levels are a direct measure of the interatomic forces at the surface. As in the bulk, the elementary vibrational excitations of a crystal surface are waves (phonons) with many different frequencies, wavelengths and propagation directions. The vibrational motion of surface atoms is determined by phonons which have their origin in the bulk of the crystal (Fig. 1a) as well as by phonons localised in the surface layer. At the surface plane, bulk phonons, with different frequencies $\omega$ and directions, project onto a single surface wave vector $\vec{Q}$ leading to bulk bands, as shown in Fig. 1b for a facecentered cubic ( $\mathrm{fcc}$ ) crystal. In addition, when a real surface is created, new modes localised in the surface plane appear as shown by dark lines in Fig. 1c. Well-defined surface modes exist only in regions of dispersion space $h \omega(Q)$ outside the surface-projected bulk bands [1]. Surface modes located inside the bulk bands usually couple to bulk phonons and thereby partially lose their localised character. These are referred to as surface "resonances". The lowest frequency surface mode, called the Rayleigh wave, is usually predominantly vertically polarised and is almost

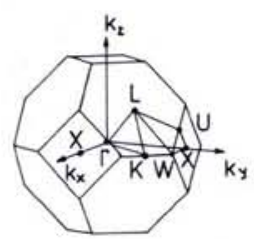

fcc 3D Brillouin zone

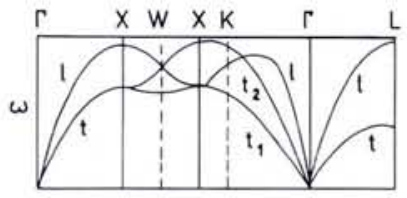

$q$ a)

Q b)

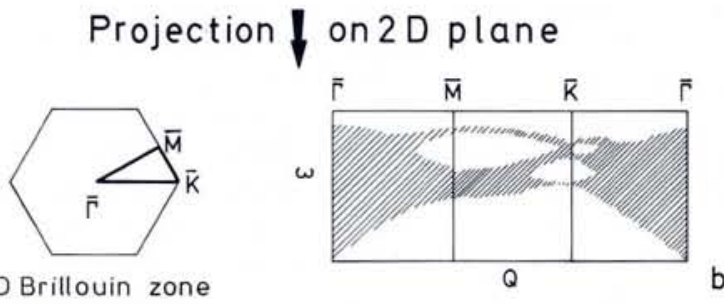

(III) 2 D Brillouin zone

Creation of surface

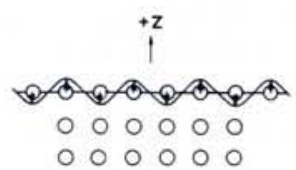

Rayleigh mode (R) at $\bar{K}$

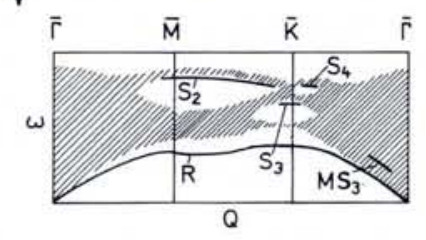

c)

Fig. 1 - Schematic diagram illustrating the heritage of the surface phonons from the bulk phonons for a face-centered cubic ( $f c c$ ) metal. The bulk dispersion curves are displayed in reciprocal lattice space in (a). Different symmetry directions from the origin at $\Gamma$ are indicated by capital letters. When projected onto the (111) surface plane the bulk phonons produce broad bands shown in (b). Due to the broken 3-d symmetry new modes appear such as the Rayleigh mode $R$ which are localised at the surface. These are indicated by the dark solid lines in (c). Throughout, small letters indicate the polarization of bulk modes (I = longitudinal, $t=$ transverse), while capital letters designate surface modes. 

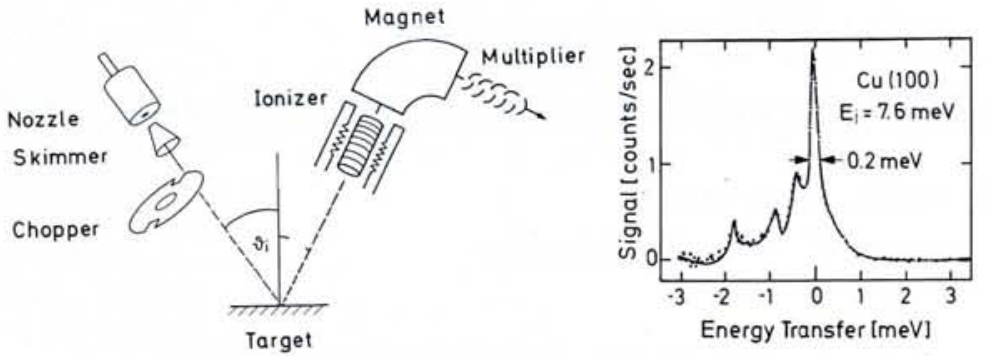

a)
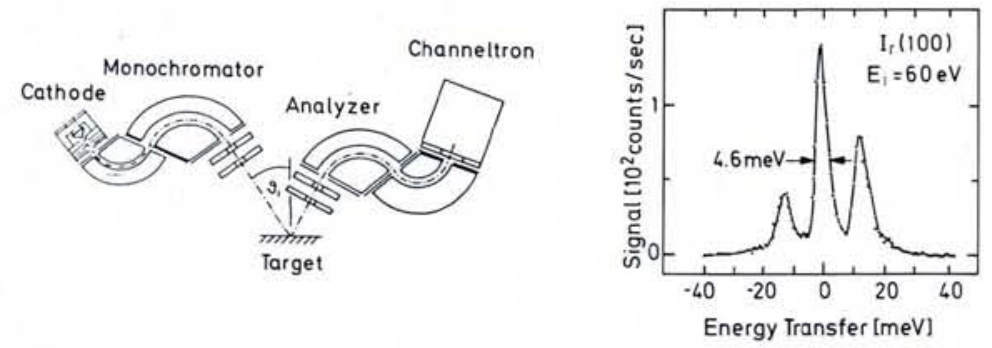

b)

Fig. 2 - Schematic diagrams showing the apparatuses used in helium atom scattering (a) and electron scattering energy transfer (b) studies of surface phonons. A typical energy transfer spectrum from recent studies of metal surfaces is shown next to each diagram. The helium atom scattering data are from [Bunjes N., Ellis J., and Witte G, private communication] and the electron scattering data from [2].

invariably observed. A well-known example of Rayleigh waves on a macroscopic scale are the long-wave components of earthquakes.

\section{Diffraction}

Measurement of one particular phonon mode requires the use of a diffraction technique, since only through diffraction is it possible to project out the momentum transferred to the individual phonons. Neutrons, which have been used with great success for measurements of bulk phonon dispersion curves, are relatively insensitive to the surface because of their small cross-sections. Nevertheless, they have been used successfully for studying surfaces. Ideal surface probes are thermal energy $\mathrm{He}$ atoms, produced by supersonic nozzle beams. Like neutrons, $\mathrm{He}$ atoms have de Broglie wavelengths commensurate with the lattice spacing and energies comparable to those of the phonons. However, in contrast to neutrons, they interact only with the surface since they are deflected by strong exchange forces at distances about $3 \AA$ above the uppermost atomic layer where the density of surface electrons is only about $10^{-3} \mathrm{e} / \AA^{3}$. Electrons also have the required de Broglie wavelengths of about $1 \AA$ but at much higher beam energies of about $200 \mathrm{eV}$. At the present time, low energy electron diffraction (LEED) is probably the most common technique for surface structure studies. Electron and helium atom surface scattering were first introduced in the late 1920's and early 1930's to demonstrate the wave nature of matter predicted by de Broglie in 1924.

\section{Experimental Techniques}

Fig. 2 shows schematic diagrams of the recently developed apparatuses now in use in the helium atom scattering (HAS) and in the electron energy loss spectroscopy (EELS) studies of surface phonons. The major breakthrough in the HAS experiments was the discovery in 1977 by Toennies and Winkelmann [3] of a dramatic narrowing of the velocity distribution of ${ }^{4} \mathrm{He}$ supersonic beam expansions. The $\mathrm{He}$ beam is produced by expanding the gas from a high pressure of about $100 \mathrm{~atm}$ through a very small orifice $(10 \mu \mathrm{m}$ diameter) into vacuum. By virtue of a quantum effect peculiar only to ${ }^{4} \mathrm{He}$ the distribution of atom velocities has a half-width of only $\Delta V / V \approx 0.5 \%$. The beam energy is directly proportional to the source temperature $T_{0}$ and is typically in the range between $40 \mathrm{meV}\left(T_{0}=160 \mathrm{~K}\right)$ and $8 \mathrm{meV}$ $\left(T_{0}=30 \mathrm{~K}\right)$ with an energy spread of about 0.40 and $0.08 \mathrm{meV}$, respectively. The scattered $\mathrm{He}$ atoms are detected by a sensitive electron bombardment ioniser and the $\mathrm{He}^{+}$ions are separated from ions of the residual gas with a mass spectrometer. By using several vacuum compartments (not shown in Fig. 2a) in the flight path it has been possible to reduce the $\mathrm{He}$ background in the ioniser to less than $10^{-15}$ torr $\left(10\right.$ atoms $\left./ \mathrm{cm}^{3}\right)$.

The ability of EELS to detect surface vibrations was first demonstrated by Probst and Piper in 1967. In these early experiments the electrons had energies of several $\mathrm{eV}$ and because of the large wavelengths, dispersion curves could not be measured. The important advance came in 1983 when Lehwald et al. [4], using tandem sector fields (see Fig. 2b), were able to obtain a resolution of $7 \mathrm{meV}$ while operating the beam at $200 \mathrm{eV}$, thus achieving a relative energy resolution of $\triangle E / E$ $\approx 2.10^{-5}$. More, recently lbach and coworkers [5] have succeeded in improving the transmission of the sector fields even further by compensating for space charge effects. Under favourable conditions an overall energy resolution of $1 \mathrm{meV}$ is achieved without sacrificing too much intensity. Helium atom and electron scattering are complementary in two important respects. He atoms interact in single collisions with the weak electron density far from the surface layer, whereas electrons, which penetrate into the crystal, also provide information on deeper lying phonon modes. High energy surface vibrations up to several $100 \mathrm{meV}$ can only be detected by EELS whereas HAS is the only technique able to resolve modes in the submeV regime.

\section{Surface Phonons of Clean Surfaces}

The first surface phonon dispersion curves were measured by HAS for the LiF(001) surface in 1981 [6]. Since then over 100 different systems have been investigated using both HAS and EELS [1]. These include all the low index surfaces of aluminium and the noble metals as well as several fcc and bcc transition metals. A large number of alkali halides, the perovskite $\mathrm{KMnF}_{3}$, several semiconductors, and several layered transition metal dichalcogenides have also been studied. Measurements are available for many systems with physi- and chemisorbed species and epitaxially grown thin films of rare-gas and metal atoms. Surface phonons have even been detected in such complex systems as the high- $\mathrm{T}_{\mathrm{c}}$ superconductors and thin films of $\mathrm{C}_{60}$ (fullerene) molecules epitaxially deposited on mica. Studies of the temperature dependence of surface phonon energies and widths provide information on phonon lifetimes. From the anomalous temperature dependence seen on $\mathrm{Fe}(110)$, striking effects of magnetic exchange forces at the surface have also been revealed.

\section{Testing calculations}

One of the ultimate goals of measuring surface phonons is to use this new data to test sophisticated first-principles calculations. Such comparisons have recently become possible for the low index aluminium surfaces for which $\mathrm{Ho}$ and Bohnen (1988) have performed total energy, frozen phonon calculations. For the same systems, Gaspar and Eguiluz (1989) have performed calculations based on an electron density response function theory which is able to predict directly the force constants between nearest and more distant neighbours. The latter theory gives particularly good results for the entire dispersion curves with only a minor adjustment of one parameter. 


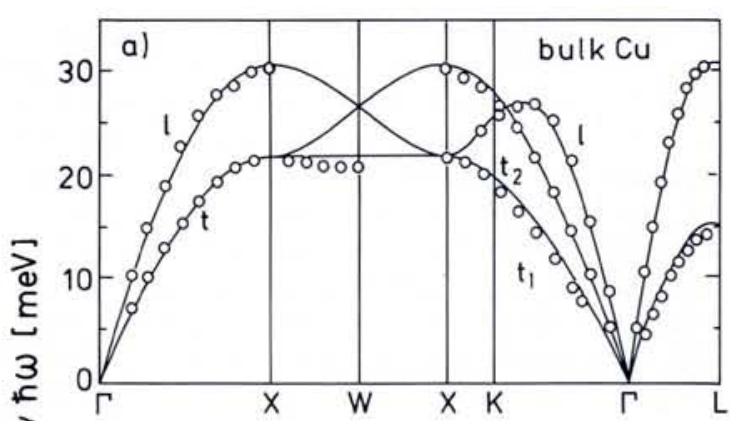

Fig. 4 - Sequence of helium atom scattering phonon dispersion curves measured on the W(001) surface [10] along the $\bar{\Gamma}-\bar{M}$ direction at: (a) 1200 $K$, well above the critical temperature $T_{c}$; (b) at $500 \mathrm{~K}$; and (c) at $220 \mathrm{~K}$, which is well below the critical transition temperature of $T_{c}=280 \mathrm{~K}$. The insets show the ball-model structures for the two phases.

Fig. 3 - (a) Comparison of neutron data for bulk phonons in copper with a one force constant model calculation. (b) A similar comparison for surface phonons on the $\mathrm{Cu}(111)$ surface measured by electron scattering (•) and $\mathrm{He}$ scattering $(0)$ with a calculation using the same force

Phonon Wave Vector $Q$

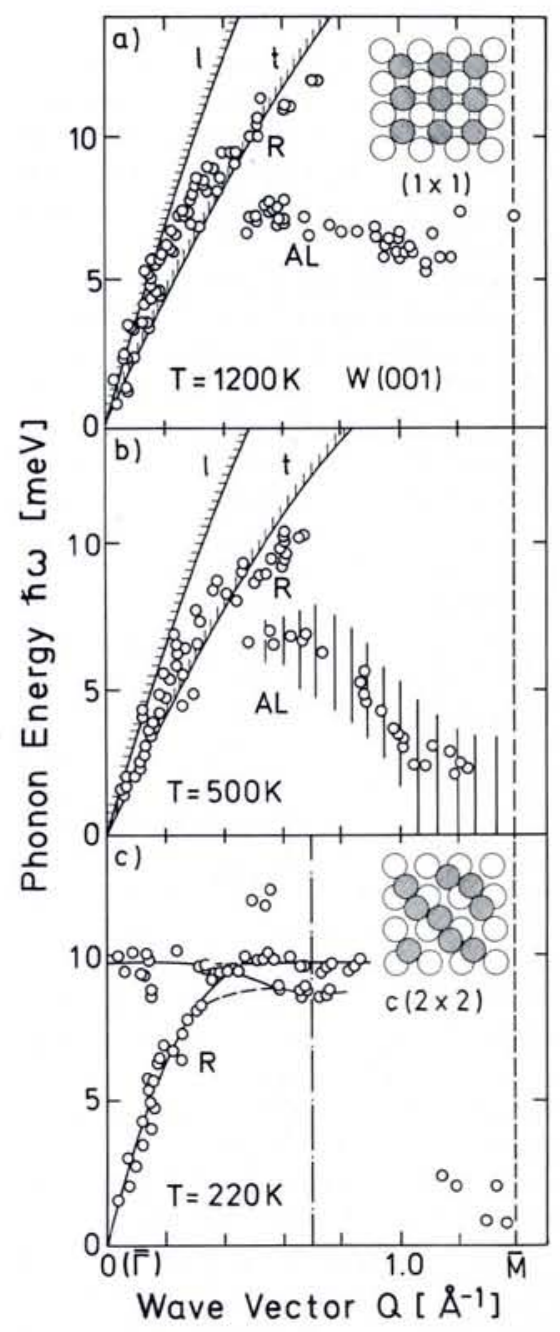

models of metal atom interactions than was previously possible from comparisons with bulk experimental data. Moreover, they provide the first evidence that atoms and molecules can also interact inelastically with the electronic degrees of freedom of a metal.

\section{W(001) surface}

One of the most extensively studied metal systems is the W(001) surface which reconstructs from a simple $(1 \times 1)$ structure to a $c(2 \times 2)$ phase when the temperature is reduced below about 280 $\mathrm{K}$. The structures in both phases have been studied by electron diffraction (LEED), $\mathrm{X}$-ray diffraction, high energy ion scattering, and core level shift spectroscopy, and are illustrated in terms of ball models in the inserts of Figs. $4 \mathrm{a}$ and c. The $\mathrm{X}$-ray experiments also revealed that $80 \%$ of the rearrangement amplitude was localised in the uppermost layer, indicating a nearly perfect two-dimensional system.

Recently, our group in Göttingen has succeeded in measuring (Fig. $4 \mathrm{a}$ ) the surface phonon dispersion curves on this system with helium atom scattering over a wide range of temperatures [9]. Even at temperatures as high as $1200 \mathrm{~K}$ the measured dispersion curves show a striking anomalous behaviour compared to other it, labelled $A L$, nor the location of the socalled "eye" mode $\mathrm{S}_{2}$ seen only by EELS. 
bcc metals such as $\mathrm{Nb}$. In W(001) there is an anomalous longitudinal mode analogous to the $A L$ resonance observed in $\mathrm{Cu}(111)$ which is shifted so strongly downward that it lies below the transverse Rayleigh mode and no longer couples to the bulk bands.

As the temperature is lowered this mode softens further and at $500 \mathrm{~K}$ approaches $h \omega=0$ at a wave vector of about $Q_{c} \approx 1.4 \AA^{-1}$ (Fig. 4b). When this happens, the lateral forces on the surface atoms vanish at interatomic distances $L=$ $2 \pi / Q_{c}$ along this particular symmetry direction. The atoms are as a result displaced and on further cooling adopt the new low-temperature structure. At $220 \mathrm{~K}$, an entirely new set of dispersion curves appears with a reduced Brillouin zone boundary consistent with this new structure.

This softening behaviour of a longitudinally polarised mode is precisely what is expected for second-order structural phase transitions according to a model first proposed by Cochran and Anderson (1960). The fact that $Q_{c}$ is incommensurate with the ion core lattice indicates the presence of a Kohn anomaly. Kohn anomalies are due to electron-phonon coupling enhanced by favourable kinematic conditions resulting from the particular shape of the Fermi surface. A strong electron-phonon coupling also leads to a periodic electronic charge modulation, called a charge density wave, as in a Wigner lattice. Thus, the surface phonon anomaly measurements in conjunction with recent photo-emission data (Kevan, 1991) reveal a charge density wave formation as the driving mechanism for the phase transition on W(001).
Charge density wave formation was suggested by Tosatti and Anderson as early as 1974. Experimental evidence for it came first from neutron and, more recently, from HAS studies of the layered transition-metal dichalcogenide compounds. They revealed that charge density waves drive the transitions between the many fascinating complex phases of these quasi-two dimensional systems. For simple metals, however, charge density waves have apparently never been observed directly, either at the surface or in the bulk. The observations made on W(001) are another striking illustration of how the reduced symmetry at the surface exposes effects not present in the bulk.

\section{Thin Films}

Recent HAS and EELS studies have concentrated on in-situ epitaxially grown thin metal films. This is a rapidly growing research area which has been stimulated by interest in superlattices and their potential applications in microelectronics. From a fundamental point of view, surface phonon studies on such systems provide a unique opportunity to follow the evolution of the interatomic forces in a metal during the transition from a two-dimensional monolayer system to the surface of a threedimensional bulk.

To avoid complications arising from the dynamical coupling of the thin film to the substrate, two different measures have been adopted. By choosing a heavy metal deposited on a light substrate, the phonons of the heavy metal appear in regions of dispersion space $h \omega(Q)$ where the substrate phonons are absent. Epitaxially grown $\mathrm{Pb}$ layers on $\mathrm{Cu}(111)$ nicely fulfill the

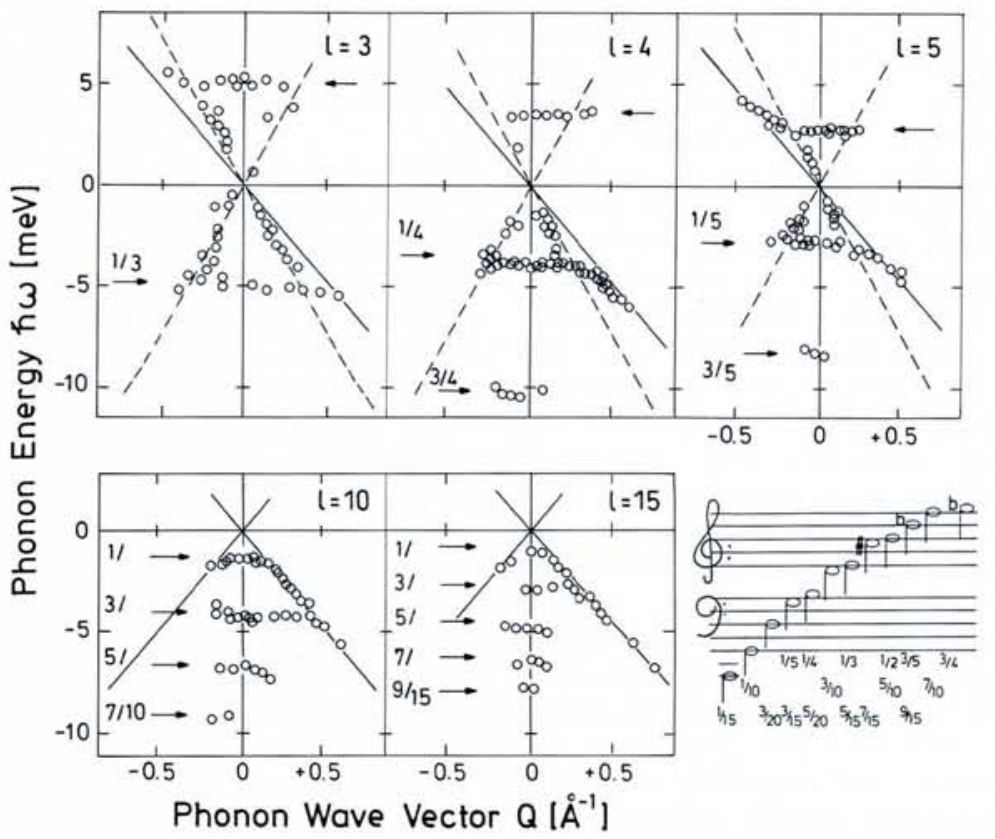

Fig. 5 - Some of the results of helium atom scattering measurements of dispersion curves of organ-pipe modes in thin $\mathrm{Na}$ films on $\mathrm{Cu}(001)$ as a function of the number of layers I. The fraction next to the arrows are equal to $(2 n-1) / l$ and are related to the wavelength of the phonon normal to the surface by $\lambda=4 / a_{11 d}(2 n-1)$. The inset at the bottom right shows all the observed frequencies on a musical scale. requirement of decoupled vibrations since the Rayleigh mode energies at the Brillouin zone boundary for $\mathrm{Cu}(111)$ are about 14 meV, which is much greater than the highest possible phonon energy of $10 \mathrm{meV}$ for $\mathrm{Pb}$. The dispersion curves for vibrations in $\mathrm{Pb}$ films of number of layers $\mathrm{I}=3$, $4,5,6,7$, and 10 layers have been measured in our laboratory using helium atom scattering. The results for $4,5,6$, and 7 layers of $\mathrm{Pb}$ deposited on $\mathrm{Cu}(111)$ reveal up to seven different modes out of a maximum of 21 observable modes, which all differ from each other. It is only after 10 layers that the dispersion curves no longer change with increasing thickness.

\section{$\mathrm{Na}$ on $\mathrm{Cu}(001)$}

Another way to avoid substrate coupling is illustrated in the case of $\mathrm{Na}$ layers on $\mathrm{Cu}(001)$. He scattering studies reveal that $\mathrm{Na}$ grows epitaxially on $\mathrm{Cu}(001)$. Because of the anomalously small elastic constants of this alkali metal, the Na phonons are decoupled from the much stiffer substrate. The layer-by-layer growth can be monitored in helium scattering by measuring the intensity undulations of the specular beam where each maximum corresponds to the completion of one filledup atomic layer. The phonon results for this system are shown in Fig. 5 [Benedek G., et al., submitted for publication]. Much to our surprise we find that the dispersion curves are nearly flat (independent of $Q$ ) except where they hybridise with the $\mathrm{Cu}$ substrate Rayleigh mode (dashed straight line) or with the Na Rayleigh mode (continuous straight line). Moreover, the frequencies of these modes change with film thickness even up to films of 20 layers. The frequencies $v$ depend on the thickness according to the formula

$$
v=\left(n^{-1 / 2} / 2 / a_{110}\right) v_{\mathrm{L}}
$$

where $n=1,2 . . . I, v_{L}$ is an effective phase velocity of a longitudinal mode propagating normal to the surface and $a_{110}$ is the interlayer spacing. An expression of the same form also holds for the frequency of the lower harmonics of an organ pipe. Therefore, the thin film vibrates like an organ pipe with an open end at the outer surface and a node at the first substrate layer. As with an open-ended organ pipe, the wavelength of the fundamental $(n=$ 1 ) is four times the thickness of the layer. Thus the $Q=0$ modes are, in fact, longitudinal modes with respect to the surface normal which have their largest amplitudes in the uppermost layer of the film.

Since the alkali metals are the simplest of all metals they are of great interest for comparisons with theory. However, they are too soft to allow the preparation of a well-defined surface from a single crystal boule. Our $\mathrm{Na}$ films experiments have made the surface phonons of the alkali metals accessible to study and have revealed a new and unexpected type of thinlayer vibration. 


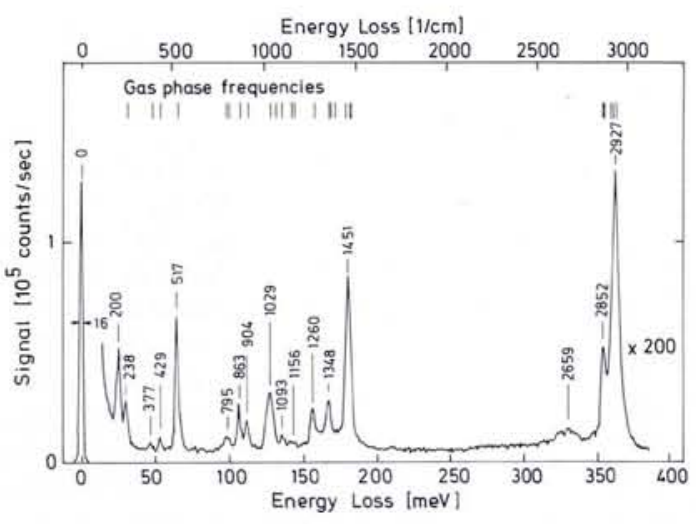

Applications to Surface Chemistry

Both spectroscopies have also provided new insight into chemical processes occurring at surfaces. EELS has long been used to study the vibrational levels of adsorbed molecules and how they differ from those of free molecules as a result of surface bonding. Moreover, the technique is able to identify and study important intermediates of reactive sequences. Fig. 6 shows a recent spectrum of cyclohexane $\mathrm{C}_{6} \mathrm{H}_{12}$ adsorbed on $\mathrm{Ni}(110)$, which is one of the most complex adsorbate systems ever studied and of great interest for catalytic dehydrogenation reactions.

The measurements were made with a new, optimised EELS apparatus which provides a resolution of $2 \mathrm{meV}\left(16 \mathrm{~cm}^{-1}\right)$ at $5 \mathrm{eV}$ beam energy [10]. Some 16 of the total number of 32 vibrational modes are resolved. The broad band around 2660 $\mathrm{cm}^{-1}$ in Fig. 6, which has no counterpart in the gas phase spectrum, results from those $\mathrm{CH}$ stretching vibrations where the hydrogen points towards the metal surface. In this situation, the hydrogen atoms move about in an environment where the density of metal electrons is appreciable. This presumably gives rise to a strong coupling of the $\mathrm{CH}$ stretching vibration to electron-hole pair excitations. Except for this particular mode, all the other frequencies of the adsorbed species agree very well with the frequencies for molecules in the gas phase. Hence the bonds within the molecule are little affected by the relatively weak van der Waals bonding to the surface.

Spectra such as the one shown in Fig. 6 provide only indirect information on the coupling of the molecule to the substrate. Additional modes, which could not be measured by other spectroscopies because of their low energies, were predicted in connection with the hindered lateral motion of molecules chemisorbed at specific sites. The chemisorption potential aids understanding of the dynamics of surface reactions, since it governs the motion of molecules along the surface.

The high resolution of helium atom scattering has made it possible for the first time to observe these low frequency

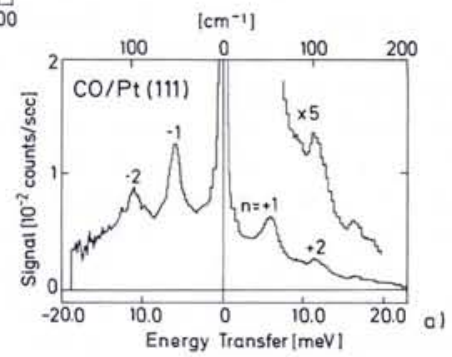

Fig. 6-EELS spectrum of $\mathrm{C}_{6} \mathrm{H}_{12}$ physisorbed on Ni(110) [10]. The electron impact energy is $5 \mathrm{eV}$ and the angles of incidence and reflection are $\approx 70^{\circ}$. The resolution is $2 \mathrm{meV}$ (FWHM). The gas phase frequencies of $\mathrm{C}_{6} \mathrm{H}_{12}$ are denoted as vertical lines at the top of the figure. The loss at 200 $\mathrm{cm}^{-1}$ is a surface resonance of the substrate.
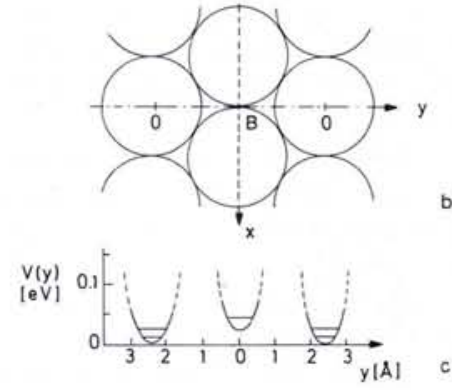

Fig. 7 - (a) Shows a typical helium atom energy loss spectrum for $10 \%$ of a monolayer of CO chemisorbed on Pt(111) revealing the frustrated translational mode on the on-top site [11]. (b) Shows the geometry of the surface. The on-top and bridge adsorption sites are indicated by $O$ and $B$, respectively. (c) Shows a cut through the potential hypersurface for a $\mathrm{CO}$ molecule interacting with the Pt(111) surface and the discrete energy levels for the frustrated translation. The measured frustrated translational modes at the on-top and bridge sites provide information on the curvature of the potential well at the two sites.

vibrational modes. Fig. 7a shows a typical helium atom energy loss spectrum obtained when scattering from a $10 \%$ monolayer of $\mathrm{CO}$ molecules adsorbed on the on-top sites on the close-packed Pt(111) surface [11]. The spectra reveal the first and second overtones of a vibrational mode, in creation and in annihilation, which is attributed to a frustrated translation of the molecule tethered to the on-top site. The frequency of this mode provides detailed information on the curvature of the potential at the on-top position, as illustrated in Fig. 7c. With increasing coverage the bridge sites are also populated, and from the observed changes in the spectra additional information on the force constants at the bridge sites is obtained. The dispersion curves of these low frequency vibrations which have been measured with both HAS, and very re- cently in EELS, also contain information on the lateral interaction of the adsorbed molecules with each other. Moreover the coupling of the adsorbate vibrations with the substrate can be inferred from the splitting of the levels at wave vectors at which the substrate and adsorbate dispersion curves intersect.

\section{Conclusions}

Helium atom and electron energy loss spectroscopies now make it possible to measure surface phonon dispersion curves of clean and adsorbate covered surfaces with a resolution of about 0.2 $\mathrm{meV}$ and $2.0 \mathrm{meV}$, respectively. Because of different couplings of the probe particle with the surface and different energy regimes, the two techniques provide complementary information.

Recent work using both techniques has concentrated on metal surfaces for which there is considerable evidence that the phonons at the surface are more sensitive to the details of the interatomic forces than bulk phonons.

Recent experiments on thin epitaxial films make it possible to follow the evolution of a metal system from a purely twodimensional adlayer system to the surface of a semi-infinite bulk. All of the different types of vibrational modes, from energies of $0.4 \mathrm{eV}$ down to $<1 \mathrm{meV}$, have now been observed for a great number of atoms and diatomic and polyatomic molecules which are physi- or chemisorbed to surfaces. In the future, with further improvements in resolution and sensitivity, even time dependent and spatially resolved studies of surface vibrations will become possible.

\section{ACKNOWLEDGEMENTS}

We are indebted to G. Benedek (Milan University) for inspiring discussions, and to $E$. Hulpke and $\mathrm{Ch}$. Ottinger for commenting on the manuscript.

\section{REFERENCES}

[1] For more extensive reviews: Surface Phonons: Eds. W. Kress and F.W. de Wette (Springer, Berlin) 1991.

[2] Lehwald S., et al., Phys. Rev. B43 (1991) 3920.

[3] Toennies J.P. and Winkelmann K., J. Chem. Phys. 66 (1977) 3965.

[4] Lehwald S., et al., Phys. Rev. Lett. 50 (1983) 518

[5] Ibach H., The Technology of High Performance Electron Energy Loss Spectrometers (Springer, Berlin) 1991.

[6] Brusdeylins G., Doak R.B. and Toennies J.P., Phys. Rev. Lett. 46 (1981) 437.

[7] Doak R.B., Harten U. and Toennies J.P., Phys. Rev. Lett. 51 (1983) 578.

[8] Jayanthi C.S., et al., Phys. Rev. Lett. 59 (1987) 795.

[9] Ernst H.J., Hulpke E. and Toennies J.P., Phys. Rev. Lett. 58 (1987) 1941; Europhys. Lett. 10 (1989) 747.

[10] Ibach H., et al., Surf. Sci., in print.

[11] Lahee A.M., Toennies J.P., and Wöll Ch., Surf. Sci. 177 (1986) 371. 